\title{
On the $D^{0}-D_{s}$ lifetime difference and $\tau \rightarrow 7 \pi+\nu_{\tau}$ decays
}

\author{
S. Nussinov ${ }^{1,2}$ and M. V. Purohit ${ }^{2}$ \\ ${ }^{1}$ School of Physics and Astronomy, \\ Tel Sackler Faculty of Exact Sciences, \\ Tel Aviv University and \\ ${ }^{2}$ Dept. of Physics 83 Astronomy, \\ Univ. of South Carolina, SC 29208
}

(Dated: October 29, 2018)

\begin{abstract}
In this paper we discuss some aspects of inclusive decays of charmed mesons and also decays of the $\tau$ lepton into $\nu_{\tau}+7 \pi$. We find that phase space effects are likely to explain the observed lifetime ratio $\tau\left(D_{s}^{+}\right) / \tau\left(D^{0}\right)=1.17$. In particular one need not appeal to a large annihilation contribution in the inclusive $D^{0}$ decay which, being absent in $D_{s}^{+}$decays could also contribute to the enhanced $D^{0}$ decay rate relative to that of the $D_{s}^{+}$. Examining a separate problem, we find that the rate for $\tau \rightarrow \nu_{\tau}+7 \pi$ is almost completely dominated by the tiny phase space for the final eight particle state. Using an effective chiral Lagrangian to estimate the matrix element yields a branching ratio into the channel of interest far smaller than the present upper bound.
\end{abstract}

PACS numbers: 13.20.Fc, 13.25.Ft, 13.35.Dx, 14.40.Lb 


\section{INTRODUCTION}

Both the (spin avaraged) square of the invariant amplitude, $\sum\left|\bar{M}_{i f}\right|^{2}$ and the Lorentz invariant phase space (LIPS) enter into the calculation of any decay or scattering process. In attempting to calculate decays of charmed particles we often find that (at least!) one of these factors is not well known. Thus for a specific decay say $D^{0} \rightarrow K \pi \pi$, or $D_{s}^{+} \rightarrow K K \pi$ the observed masses of the final and initial particles entering the LIPS are precisely known. On the other hand the relatively low energies of the final hadrons $O(M / 3=0.6 \mathrm{GeV})$, precludes any perturbative approach to the calculation of the decay matrix ellement.

For inclusive (semi- or non-leptonic) decays a perturbative approach is used. In the $\Lambda_{\mathrm{QCD}} / M_{Q} \rightarrow 0$ and $/$ or $F_{\mathrm{D}, \mathrm{B}} / M_{\mathrm{D}, \mathrm{B}} \rightarrow 0$ limits we neglect the spectator quark and view the process as the decay of a "free" heavy quark: $Q \rightarrow q \bar{l} \nu$ or $Q \rightarrow q u \bar{d}$, with $Q=c$ and $q=s$ for the CKM favored decays. One uses here the "Hadron Quark Duality" assumption that for the inclusive decay of interest, hadronization generates just a "Final State Interaction Phase". This "Phase" is a unitary matrix which shuffles decay probabilities between different channels without modifying inclusive rates.

The four-quark "Effective Lagrangian" describing the $Q$ decay includes QCD normalization effects due to integration over momenta between the high $W$ mass and the intermediate $M_{Q}$ scale. Unlike the ultimate "Renormalization" yielding a "Chiral Effective Lagrangian" appropriate for scales of $\Lambda_{\mathrm{QCD}} \approx 300 \mathrm{MeV}$ (and which would then directly yield the amplitudes for specific hadronic decays), the effective 4-quark Lagrangian can be estimated via perturbative QCD. The resultant enhancement of the ordinary $c \bar{s} \bar{d} u$ term and the negative interference (for the $\mathrm{D}$ decays) with the new $c \overline{u s} d$ term helps explain the reduction of the semileptonic branchings and the $\Gamma\left(D^{0}\right) / \Gamma\left(D^{+}\right) \approx 2$ width ratio.

The latter could in part be due to annihilation with the spectator $\bar{u}$ quark which is present in the $D^{0}$ but not in the $D^{+}$. While such annihilation transcends the spectator model it is suppressed by a $F_{q} / M_{q}$ factor.

However the inclusive calculation of actual rates (rather than ratios thereof) includes also as essentially a phase space factor: the $M_{Q}^{5} f(r)$ factor with $r=m / M=0.1--0.07$. Neither the charm nor the strange quark masses relevant for this decay are independently measured and even a $2-3 \%$ variation of $M$ can cause a 10-15\% change in the actual decay rate.

Recent experiments which measure the $D_{s}^{+}$lifetime with $3 \%$ precision suggest that it is 
longer than the $D^{0}$ lifetime by $\sim 17 \%$. This modest difference can be due, in particular, to the following two distinct effects:

1. The above mentioned annihilation amplitude contributing to the $D^{0}$ width which is practically absent in $D_{s}$ decays due to a large helicity $\left(m_{u} / m_{c}\right)^{2}$ suppression of the final multipion states.[1]

2. Due to the helicity suppression of decays into multipions, the final states in $D_{s}^{+}$decays contain a $K$ and a $\bar{K}$ rather than a $K$ and a $\pi$ as in the "corresponding" D decay, e.g., $D_{s}^{+} \rightarrow K^{+} K^{-} \pi^{+}$versus $D^{0} \rightarrow \bar{K}^{0} \pi^{+} \pi^{-}$. This, in turn, may reduce the corresponding phase space.

Elaborating on point 2 we note that the $D_{s}^{+}-D^{0}$ mass difference $(=104 \mathrm{MeV})$, is the smallest of the mass differences between strange and corresponding non-strange mesons and baryons:

$$
\begin{aligned}
m_{K}^{+}-m_{\pi}^{+} & =354.1 \mathrm{MeV} \\
m_{K^{*+}}-m_{\rho}^{+} & =122.4 \mathrm{MeV} \\
m_{\Lambda}-m_{n} & =176.1 \mathrm{MeV}
\end{aligned}
$$

Hence phase space effects in the actual physical final multiparticle states, for intermediate resonances, and even within the spectator model, reduce $\Gamma\left(D_{s}^{+}\right) / \Gamma\left(D^{0}\right)$. Conceivably this could account for the deviation of this ratio from unity.

In the second half of the paper we consider the decay of the tau lepton into final states with many pions. This is motivated by the fact that strong upper bounds of $1.8 \times 10^{-5}$ and $3 \times 10^{-6}$ have been obtained for the branching ratio into the state with seven charged pions. We find that this decay rate is almost completely dominated by the decrease in phase space as the energy per final state pion is reduced. We use the simplest chiral Lagrangian to estimate the relevant matrix elements and decay rate. The estimated branching ratios are far smaller than the above upper bounds - making the observation of these decays extremely unlikely. We argue that despite the existence of many resonances in the 3 and 4 pion channels the utilization of an effective local Lagrangian approach is indeed justified. The condition for this is that the resonances are broad as they are in the case of interest. We 
also address possible unusual "collective" enhancements which could occur for a sufficiently large number of pions. We find however that for the case at hand with only seven pions no such enhancement is likely to occur.

\section{LIPS FOR SPECIFIC HADRONIC DECAYS}

We have examined in detail the difference in decay rates of the $D^{0}$ and the $D_{s}^{+}$due to phase space. We began by taking the entire list of $D^{0}$ decay modes from the Particle Data Group (PDG)[2]. The sum of the $D^{0}$ branching ratios listed there is approximately $122 \%$ where the excess is due to quantum interference. This indicates that measurements of the $D^{0}$ decay modes are substantially complete and can be used to estimate the total $D_{s}^{+}$rate if we assume that for each $D^{0}$ decay mode there is a corresponding $D_{s}^{+}$decay mode (see table I below) and that the corresponding partial widths are simply the square of a common matrix element times the phase space of each decay. We thus compute the expected partial widths for the $D_{s}^{+}$decay modes corresponding to each $D^{0}$ decay mode and sum to obtain the total decay rate.

While this procedure may not be precise for individual decay modes due to final state interactions, it should correctly approximate the phase space effect when we sum over all the decay modes. In implementing the procedure we took into account the variation of phase space due to width of resonances. When converting $s \bar{s}$ final states to $\eta$ and $\eta^{\prime}$ we used the simplest mixing described in the PDG.[2] Similarly, we followed the PDG in assuming that the $K_{1 A}$ is an equal mixture of $K_{1}(1270)$ and $K_{1}(1400)$. For two-body decays only, we applied an extra factor of $p^{* 2}$ if the decay products were a vector and a pseudoscalar. (Since this tends to reduce the phase space effect it is clearly conservative). The widths of the two purely leptonic decay modes of the $D_{s}^{+}$were added to the sum (a $1.2 \%$ correction). Finally,

we ignored the $K^{0} \bar{K}^{0}$ and the $K_{S}^{0} K_{S}^{0} K_{S}^{0} K_{S}^{0}$ decay modes of the $D^{0}$ since the analogues in $D_{s}^{+}$decay are hard to identify. Again, these decay modes contribute well under $1 \%$ of the total $D^{0}$ width.

The result of this exercise is shown at the bottom of table I. The $D^{0}$ width is larger than the estimated $D_{s}^{+}$width, predicting that the $D_{s}^{+}$lifetime will be longer than the $D^{0}$ lifetime by about $25 \%$. This is to be compared to the measured difference which is about $17 \%$. Thus it may well be that the complete $D_{s}^{+}-D^{0}$ lifetime difference is due merely to phase space 
effects and there is no need to invoke any appreciable additional annihilation contribution to the $D^{0}$ inclusive decay rate. 
TABLE I: In this table we list the phase space for $D^{0}$ and corresponding $D_{s}^{+}$decays, the $D^{0}$ width from the particle tables and finally, the estimated $D_{s}^{+}$width.

\begin{tabular}{|c|c|c|c|c|c|}
\hline$D^{0}$ Decay & $D_{s}^{+}$Decay & $D^{0}$ Phase & $D_{s}^{+}$Phase & $D^{0}$ & $D_{s}^{+}$Estimated \\
\hline Mode & Mode & Space & Space & $\begin{array}{l}\text { Partial Width } \\
\qquad\left(p s^{-1}\right)\end{array}$ & $\begin{array}{l}\text { Partial Width } \\
\qquad\left(p s^{-1}\right)\end{array}$ \\
\hline$\mu^{+} \nu_{\mu}$ & $\mu^{+} \nu_{\mu}$ & 1.56575 & 1.56627 & 0.00000 & 0.00927 \\
\hline$\tau^{+} \nu_{\tau}$ & $\tau^{+} \nu_{\tau}$ & 0.14393 & 0.29084 & 0.00000 & 0.01411 \\
\hline$K^{-} e^{+} \nu_{e}$ & $\eta e^{+} \nu_{e}$ & 1.96132 & 1.42768 & 0.08822 & 0.06422 \\
\hline$K^{-} \mu^{+} \nu_{\mu}$ & $\eta \mu^{+} \nu_{\mu}$ & 1.91829 & 1.39347 & 0.07804 & 0.05669 \\
\hline$K^{-} \pi^{0} e^{+} \nu_{e}(N R)$ & $\eta \pi^{0} e^{+} \nu_{e}$ & 0.87300 & 0.58321 & 0.02254 & 0.01506 \\
\hline $\bar{K}^{0} \pi^{-} e^{+} \nu_{e}(N R)$ & $\bar{K}^{0} K^{0} e^{+} \nu_{e}$ & 0.85393 & 0.45099 & 0.03514 & 0.01856 \\
\hline$K^{*-} e^{+} \nu_{e}$ & $\phi e^{+} \nu_{e}$ & 0.74673 & 0.66038 & 0.04896 & 0.04330 \\
\hline$\pi^{-} e^{+} \nu_{e}$ & $K^{0} e^{+} \nu_{e}$ & 3.59061 & 2.28553 & 0.00897 & 0.00571 \\
\hline$K^{-} \pi^{0} \mu^{+} \nu_{\mu}(N R)$ & $\eta \pi^{0} \mu^{+} \nu_{\mu}$ & 0.75653 & 0.50155 & 0.02254 & 0.01494 \\
\hline $\bar{K}^{0} \pi^{-} \mu^{+} \nu_{\mu}(N R)$ & $\bar{K}^{0} K^{0} \mu^{+} \nu_{\mu}$ & 0.73938 & 0.38250 & 0.03514 & 0.01818 \\
\hline$K^{*-} \mu^{+} \nu_{\mu}$ & $\phi \mu^{+} \nu_{\mu}$ & 0.71949 & 0.63552 & 0.04896 & 0.04324 \\
\hline$\pi^{-} \mu^{+} \nu_{\mu}$ & $K^{0} \mu^{+} \nu_{\mu}$ & 3.51730 & 2.24131 & 0.00897 & 0.00571 \\
\hline$K^{-} \pi^{+}$ & $\eta \pi^{+}$ & 1.45053 & 1.31153 & 0.09283 & 0.08393 \\
\hline $\bar{K}^{0} \pi^{0}$ & $K^{+} \bar{K}^{0}$ & 1.44938 & 1.35708 & 0.05114 & 0.04788 \\
\hline $\bar{K}^{0} \pi^{+} \pi^{-}(N R)$ & $K^{+} K^{-} \pi^{+}(N R)$ & 1.67444 & 1.31914 & 0.03563 & 0.02807 \\
\hline$K^{-} \pi^{+} \pi^{0}(N R)$ & $K^{-} K^{+} \pi^{+}$ & 1.69923 & 1.31914 & 0.01672 & 0.01298 \\
\hline $\bar{K}^{0} \pi^{0} \pi^{0}(N R)$ & $\bar{K}^{0} K^{+} \pi^{0}$ & 1.68900 & 1.31547 & 0.01890 & 0.01472 \\
\hline$K^{-} \pi^{+} \pi^{+} \pi^{-}(N R)$ & $K^{-} K^{0} \pi^{+} \pi^{+}$ & 0.53702 & 0.24755 & 0.04217 & 0.01944 \\
\hline $\bar{K}^{0} \pi^{+} \pi^{-} \pi^{0}(N R)$ & $\bar{K}^{0} K^{+} \pi^{+} \pi^{-}$ & 0.53483 & 0.24755 & 0.05090 & 0.02356 \\
\hline$K^{-} \pi^{+} \pi^{0} \pi^{0}$ & $K^{-} K^{+} \pi^{0} \pi^{+}$ & 0.55103 & 0.25678 & 0.36355 & 0.16941 \\
\hline$K^{-} \pi^{+} \pi^{+} \pi^{-} \pi^{0}(N R)$ & $K^{-} K^{0} \pi^{+} \pi^{+} \pi^{0}$ & 0.05758 & 0.01268 & 0.00242 & 0.00053 \\
\hline $\bar{K}^{0} \pi^{+} \pi^{+} \pi^{-} \pi^{-}$ & $\bar{K}^{0} K^{+} \pi^{+} \pi^{-} \pi^{0}$ & 0.05473 & 0.01268 & 0.01406 & 0.00326 \\
\hline $\bar{K}^{0} \pi^{+} \pi^{-} \pi^{0} \pi^{0}$ & $\bar{K}^{0} K^{+} \pi^{0} \pi^{0} \pi^{0}$ & 0.05734 & 0.01354 & 0.25691 & 0.06068 \\
\hline
\end{tabular}




\begin{tabular}{|c|c|c|c|c|c|}
\hline $\bar{K}^{0} K^{+} K^{-}(N R)$ & $\eta \bar{K}^{0} K^{+}$ & 0.31535 & 0.19602 & 0.01236 & 0.00768 \\
\hline$K^{-} K^{+} K^{-} \pi^{+}$ & $\eta K^{+} K^{-} \pi^{+}$ & 0.00769 & 0.00726 & 0.00051 & 0.00048 \\
\hline$K^{-} K^{+} \bar{K}^{0} \pi^{0}$ & $\eta K^{+} \bar{K}^{0} \pi^{0}$ & 0.00769 & 0.00725 & 0.01745 & 0.01646 \\
\hline $\bar{K}^{0} \eta$ & $\bar{K}^{0} K^{+}$ & 1.30045 & 1.35708 & 0.01697 & 0.01770 \\
\hline $\bar{K}^{0} \rho^{0}$ & $K^{*+} \bar{K}^{0}$ & 1.13230 & 1.08952 & 0.02933 & 0.02912 \\
\hline$K^{-} \rho^{+}$ & $\eta \rho^{+}$ & 1.13507 & 0.93361 & 0.26175 & 0.16237 \\
\hline $\bar{K}^{0} \omega$ & $\bar{K}^{0} K^{*+}$ & 1.12856 & 1.08952 & 0.05090 & 0.05105 \\
\hline $\bar{K}^{0} \eta^{\prime-}(958)$ & $\bar{K}^{0} K^{+}$ & 0.95141 & 1.35708 & 0.04144 & 0.05912 \\
\hline $\bar{K}^{0} f_{0}(980)$ & $\bar{K}^{0} K^{* 0}(1430)^{+}$ & 0.92292 & 0.33782 & 0.01381 & 0.00506 \\
\hline $\bar{K}^{0} \phi$ & $\bar{K}^{0} K^{*+}$ & 0.87666 & 1.08952 & 0.02084 & 0.04460 \\
\hline$K^{-} a 1(1260)$ & $\bar{K}^{0} K_{1 A}^{+}$ & 0.51031 & 0.47496 & 0.17693 & 0.15902 \\
\hline $\bar{K}^{0} f_{2}(1270)$ & $\bar{K}^{0} K_{2}^{*}(1430)^{+}$ & 0.40873 & 0.27454 & 0.00994 & 0.00667 \\
\hline $\bar{K}^{0} f_{0}(1370)$ & $\bar{K}^{0} K_{0}^{*}(1430)^{+}$ & 0.28812 & 0.33782 & 0.01672 & 0.01961 \\
\hline$K^{*-} \pi^{+}$ & $\phi \pi^{+}$ & 1.19686 & 1.13584 & 0.12118 & 0.11546 \\
\hline $\bar{K}^{* 0} \pi^{0}$ & $\bar{K}^{* 0} K^{+}$ & 1.19413 & 1.08819 & 0.07513 & 0.06338 \\
\hline $\bar{K}^{* 0} \pi^{+} \pi^{-}$ & $\phi \pi^{+} \pi^{0}(N R)$ & 0.55761 & 0.49927 & 0.05332 & 0.04774 \\
\hline$K^{-} \pi^{+} \rho^{0}$ & $\eta \pi^{+} \rho^{0}$ & 0.27779 & 0.17306 & 0.15269 & 0.09513 \\
\hline$K^{*-} \rho^{+}$ & $\phi \rho^{+}$ & 0.67368 & 0.61124 & 0.14784 & 0.13414 \\
\hline$K 1(1270)^{-} \pi^{+}$ & $f_{1}(1285) \pi^{+}$ & 0.81186 & 0.88490 & 0.02569 & 0.03709 \\
\hline$K_{0}^{*}(1430)^{-} \pi^{+}$ & $f_{0}(980) \pi^{+}$ & 0.63668 & 1.16733 & 0.02521 & 0.04621 \\
\hline $\bar{K}^{* 0} \eta$ & $\bar{K}^{* 0} K^{+}$ & 0.97557 & 1.08819 & 0.04605 & 0.07125 \\
\hline$K^{-} \pi^{+} \omega(N R)$ & $\eta \pi^{+} \omega$ & 0.23732 & 0.14638 & 0.04605 & 0.02840 \\
\hline $\bar{K}^{* 0} \omega$ & $K^{*+} \bar{K}^{* 0}$ & 0.67860 & 0.65378 & 0.02666 & 0.02569 \\
\hline$K^{-} \pi^{+} \eta^{-}$ & $\eta \pi^{+} \eta^{-}$ & 0.06989 & 0.04908 & 0.01697 & 0.01192 \\
\hline$\pi^{+} \pi^{-}$ & $\pi^{+} K^{0}$ & 1.55309 & 1.46143 & 0.00368 & 0.00347 \\
\hline$\pi^{0} \pi^{0}$ & $\pi^{0} K^{+}$ & 1.55424 & 1.46363 & 0.00204 & 0.00192 \\
\hline$\pi^{+} \pi^{-} \pi^{0}$ & $\pi^{+} K^{+} \pi^{-}$ & 3.23927 & 2.01929 & 0.03878 & 0.02417 \\
\hline$\pi^{+} \pi^{-} \pi^{+} \pi^{-}$ & $\pi^{+} K^{+} \pi^{0} \pi^{-}$ & 1.87461 & 0.79452 & 0.01769 & 0.00750 \\
\hline$\pi^{+} \pi^{-} \pi^{+} \pi^{-} \pi^{0}$ & $\pi^{+} K^{+} \pi^{+} \pi^{-} \pi^{-}$ & 0.41812 & 0.10642 & 0.04605 & 0.01172 \\
\hline$\pi^{+} \pi^{-} \pi^{+} \pi^{-} \pi^{+} \pi^{-}$ & $\pi^{+} K^{+} \pi^{+} \pi^{-} \pi^{-} \pi^{0}$ & 0.03757 & 0.00537 & 0.00097 & 0.00014 \\
\hline
\end{tabular}




\begin{tabular}{cccccc}
$K^{+} K^{-}$ & $\eta K^{+}$ & 1.33247 & 1.18007 & 0.01030 & 0.00912 \\
$K^{0} K^{-} \pi^{+}(N R)$ & $\eta K^{0} \pi^{+}$ & 1.01935 & 0.68613 & 0.00557 & 0.00375 \\
$\bar{K}^{0} K^{+} \pi^{-}(N R)$ & $K^{0} \bar{K}^{0} K^{+}$ & 1.01935 & 0.49496 & 0.00921 & 0.00447 \\
$K^{+} K^{-} \pi^{0}$ & $\pi^{+} \pi^{-} \pi^{+}$ & 1.03921 & 3.67230 & 0.03151 & 0.11134 \\
$K^{+} K^{-} \rho^{0}$ & $K^{+} K^{-} K^{*+}$ & 0.04758 & 0.01903 & 0.00218 & 0.00087 \\
$K^{0} \bar{K}^{0} \pi^{+} \pi^{-}$ & $\pi^{+} K^{0} \bar{K}^{0} K^{0}$ & 0.13646 & 0.01137 & 0.01648 & 0.00137 \\
$K^{+} K^{-} \pi^{+} \pi^{-} \pi^{0}$ & $K^{+} K^{-} \pi^{+} \pi^{-} K^{+}$ & 0.00464 & 0.00009 & 0.00751 & 0.00015 \\
$K^{*+} K^{-}$ & $\eta K^{*+}$ & 1.02605 & 0.78911 & 0.00848 & 0.00430 \\
$K^{*-} K^{+}$ & $\phi K^{+}$ & 1.02605 & 0.96836 & 0.00436 & 0.00409 \\
$\phi \rho^{0}(N R)$ & $\phi K^{*+}$ & 0.39711 & 0.35351 & 0.00145 & 0.00129 \\
$\phi \pi^{+} \pi^{-}(N R)$ & $\phi K^{+} \pi^{0}$ & 0.34522 & 0.17350 & 0.00170 & 0.00085 \\
$\bar{K}^{* 0} K^{* 0}$ & $\phi K^{*+}$ & 0.41460 & 0.35351 & 0.00339 & 0.00289 \\
\hline Total & Total & & & 2.95982 & 2.22225 \\
\hline
\end{tabular}




\section{REDUCED $D_{s}^{+}$INCLUSIVE DECAY RATE VIA A REDUCED EFFECTIVE CHARM QUARK MASS}

Phase space effects manifest also via the effective mass of the decaying $c$ quark. The binding of the $c$ quark is stronger in the $c \bar{s}$ system then in the $c \bar{u}$ system. Since the fifth power of this mass enters in $\Gamma(Q \rightarrow q l \nu)$ small shifts of $m(Q)$ may have enhanced, perceptible

effects in the spectator model. This is analogous [3] to the reduction due to the Coulombic binding $\left(Z \alpha^{2} / 2\right) m_{\mu}$ of the $\mu^{-} \rightarrow e^{-} \nu_{\mu} \bar{\nu}_{e}$ decay rate in muonic atoms. The asymptotic physical state after the $\mu^{-}$decay no longer has Coulomb binding. Consequently the effective mass of the decaying muon and the corresponding phase space and decay rate will be reduced by $1-r=\left(1-\mathrm{BE} / m_{\mu}\right)$ and by $(1-r)^{5}$ respectively, where BE is the binding energy of the muon.

The present case is not as clear cut. Due to confinement the spectator quark of the initial $D$ "bound state" and the three quarks from the decay of the $c$ quark appear (albeit in new rearranged forms) as constituents of the final hadrons.

Thus color binding is not completely lost here. This is in contradistinction with the loss of Coulombic binding in the above $\mu^{-}$decay. Rather we have here only an enhanced binding of the $c \bar{s}$ (or $D_{s}^{+}$) system as compared with the $c \bar{u}$ or $D^{0}$ system. This "relatively" low $m_{D_{s}^{+}}$ mass is indeed the source of the phase space reduction in the actual physical decay channels of the $D_{s}^{+}$relative to that of the $D^{0}$ which is precisely what has been discussed in section II. Hence, whatever "effect" is eventually found in the present section, does not add up to the above suppression. Rather, it is just another way, utilizing quark hadron duality, of restating the same effect or part thereof. In the spirit of the spectator model of the decay we consider only the reduction due to tighter binding of the $c$ quark mass in $D_{s}^{+}=c \bar{s}$ as compared with that of the $c$ quark inside $D^{0}=c \bar{u}$. Since binding effects are often deemed to be pertinent only to the interacting two body system as a whole i.e., to the $c$ and $\bar{q}=\bar{s}$ (or $\bar{u}$ ) the separation of the "charm quark associated part" is, at best, ambiguous.

If the $Q \bar{q}$ system was naively treated as a non-relativistic Coulomb two-body system then the binding energy $B E=\frac{\alpha_{S}^{2}}{2} m_{Q, q}$ where the reduced mass $m_{Q, q}$ is given by

$$
m_{Q, q}=\frac{m_{q} m(Q)}{m_{Q}+m_{q}}
$$

Using $m_{c}=1.5 \mathrm{GeV}, m_{u}=0.3 \mathrm{GeV}$ and $m_{s}=0.45 \mathrm{GeV}$, we find that the ratio of reduced 
masses in the $D_{s}^{+}$and $D^{0}$ system is about 0.8 and their difference is of order $100 \mathrm{MeV}$. The Coulombic binding difference between $D_{s}^{+}$and $D^{0}$ is then $B E=\left(\alpha_{S}^{2} / 2\right) 100 \mathrm{MeV}=50 \mathrm{MeV}$, where we arbitrarily used $\alpha_{S}=1$. If half of this is assigned to the charmed quark then its effective mass in the $D_{s}^{+}$decay will be $25 / 1500=1.6 \%$ lower than that in $D^{0}$ decays. The resulting phase space reduction due to $m_{c}^{5}$ is then $8 \%$. The Coulombic interaction maximizes the effect of quark (reduced) mass changes, as in this case the reduced quark mass $m_{Q, q}$ is the only relevant parameter of mass dimension entering the problem. Once other-dimensional parameters are introduced this dependence on $m_{Q, q}$ gets diluted and weakened. In particular this is the case for any effective interaction

$$
V(r)=r^{\beta} \quad \text { with } \quad 1>\beta>-1
$$

Such potentials may indeed be more appropriate to describe the $Q \bar{q}$ interaction at the $\sim 0.5 \mathrm{fm}$ scale of the physical bound state. Thus the spectator model phase space effect is smaller than $8 \%$. It is therefore unlikely to account, by itself, for the observed $17 \%$ reduction in $\Gamma\left(D_{s}^{+}\right)$when compared to $\Gamma\left(D^{0}\right)$.

\section{MULTIPLE PION STATES IN THE DECAY OF THE TAU LEPTON}

Upper bounds on the mass of the tau neutrino were deduced from analysis of the decay $\tau^{-} \rightarrow \pi^{+} \pi^{+} \pi^{-} \pi^{-} \pi^{-} \nu_{\tau}$. The seven pion mode, $\tau^{-} \rightarrow \pi^{+} \pi^{+} \pi^{+} \pi^{-} \pi^{-} \pi^{-} \pi^{-} \nu_{\tau}$ if appreciable,

could lead to a more stringent bound. The OPAL and CLEO collaborations searched for these decays. Finding none they deduced the following upper bounds

$$
B R\left(\tau^{-} \rightarrow \pi^{+} \pi^{+} \pi^{+} \pi^{-} \pi^{-} \pi^{-} \pi^{-} \nu_{\tau}\right)<1.8 \times 10^{-5} \quad \text { (OPAL) }
$$

and

$$
B R\left(\tau^{-} \rightarrow \pi^{+} \pi^{+} \pi^{+} \pi^{-} \pi^{-} \pi^{-} \pi^{-} \nu_{\tau}\right)<2.6 \times 10^{-6} \quad \text { (CLEO) }
$$

at the $95 \%$ CL.

These rare tau decays are intersting in a purely hadronic context as well. Many "soft" pions are produced with small $(\mathrm{L}=0$ or $\mathrm{L}=1)$ overall angular momenta. These decays may 
provide a simpler setting than $p \bar{p}$ annihilations at rest for studying such systems. Here, but not in the annihilation, the pions are produced via the local vector/axial currents. This in particular allows us to estimate the expected rates via chiral Lagrangians [4]. We find, mainly due to phase space limitations, that the expected rate is much smaller than the existing bounds. Thus finding even ONE unambiguous such decay would indicate strong "Dynamical" enhancements. The latter could in particular indicate the onset of non-perturbative "Condensate" effects operative only for a sufficiently large number of pions.

In order to estimate the decay rate $\Gamma\left(\tau^{-} \rightarrow \pi^{+} \pi^{+} \pi^{+} \pi^{-} \pi^{-} \pi^{-} \pi^{-} \nu_{\tau}\right)$ we utilize the simplest chiral Lagrangian

$$
\mathcal{L}=2 f_{\pi}^{2}\left(\exp \left(\frac{i \hat{\phi}}{2 f_{\pi}}\right) \stackrel{\leftrightarrow}{\partial}_{\mu} \exp \left(\frac{-i \hat{\phi}}{2 f_{\pi}}\right)\right)^{2}
$$

with

$$
\hat{\phi}=\phi_{i} \tau_{i}
$$

and $f_{\pi} \approx 90 \mathrm{MeV}$.

The corresponding Noether current:

$$
\mathcal{J}_{\mu}=\frac{\partial}{\partial_{\mu} \phi} \mathcal{L}=2 f_{\pi}^{2}\left(\exp \left(\frac{i \hat{\phi}}{2 f_{\pi}}\right) \stackrel{\leftrightarrow}{\partial}_{\mu} \exp \left(\frac{-i \hat{\phi}}{2 f_{\pi}}\right)\right)
$$

yields the vector / axial currents as its $\hat{\phi}$ even / odd parts. We can, as indicated in Appendix I, extract the seventh order term

$$
c_{7}\left(\partial_{\mu} \hat{\phi}\right) \frac{(\hat{\phi} \cdot \hat{\phi})^{3}}{\left(2 f_{\pi}\right)^{5}}
$$

relevant to the $\left\langle 0\left|J_{\mu}^{A}(x)\right| \pi_{1} \pi_{2} \ldots \pi_{7}\right\rangle$ amplitude of interest.

Had we started from a more elaborate Lagrangian we might have generated $J_{A}^{\mu}(x)$ with extra derivatives. However in the "Soft pion" limit - which is almost achieved here - the minimal form of equation [11] with just one derivative may dominate.

The complete effective Lagrangian for the tau decay also contains the lepton current:

$$
\mathcal{L}=\frac{G_{F}}{\sqrt{2}} \int d^{4} x \bar{\Psi}_{\tau}(x) \gamma_{\mu} \gamma_{5} \Psi_{\nu}(x) c_{7}\left(\partial_{\mu} \phi\right) \phi^{6}
$$


using $\partial_{\mu} \phi^{7} \approx 7\left(\partial_{\mu} \phi\right) \phi^{6}, \not \partial \Psi_{\tau}(x)=m_{\tau} \Psi_{\tau}(x), \not \partial \Psi_{\nu}(x)=0, \gamma_{5} \Psi_{\nu}=\Psi_{\nu}$ and intergrating by parts we can rewrite the decay Lagrangian as:

$$
\mathcal{L}^{\mathrm{eff}}=\frac{c_{7}}{7} \frac{G_{f}}{\left(2 f_{\pi}\right)^{5}} m_{\tau} \Psi_{\tau}(x) \Psi_{\nu_{\tau}}(x) \phi^{7}(x)
$$

In momentum space $\bar{\Psi}_{\tau} \gamma_{5} \Psi_{\nu}$ becomes $\bar{u}_{\tau}(p) u_{\nu}(q)$ with $p, q$ the four momenta of the tau and tau neutrino. Upon squaring and spin summation this yields an extra $m(\tau)$ factor (the massless tau neutrino is conventionally normalized to $\bar{u} u=1$ ). Thus the Lagrangian (13) yields:

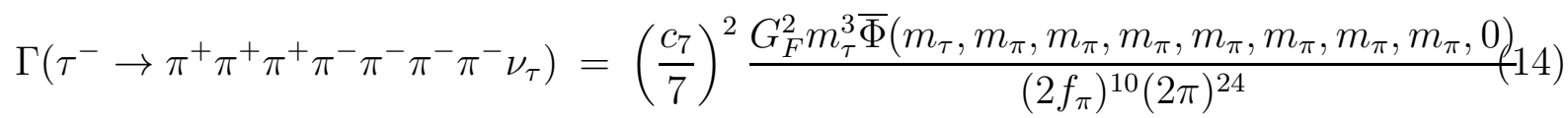

with $\bar{\Phi}$ being the eight body phase space for the $\tau^{-} \rightarrow \pi^{+} \pi^{+} \pi^{+} \pi^{-} \pi^{-} \pi^{-} \pi^{-} \nu_{\tau}$ decay.

The above phase space and for the other tau decays into states with $n$ final pions are evaluated via the composition formula

$$
\Phi_{\left(n_{1}+n_{2}\right)}(W)=\prod \int_{\left(n_{1} m_{\pi}\right)^{2}}^{\infty} d W_{1}^{2} \int_{\left(n_{2} m_{\pi}\right)^{2}}^{\infty} d W_{2}^{2} \frac{\Delta^{1 / 2}\left(W, W_{1}, W_{2}\right)}{2 W^{2}} \Phi_{n_{1}}\left(W_{1}\right) \Phi_{n_{2}}\left(W_{2}\right) \Theta(\Delta(15)
$$

where

$$
\Delta\left(W, W_{1}, W_{2}\right)=\left[W^{2}-\left(W_{1}+W_{2}\right)^{2}\right]\left[W^{2}-\left(W_{1}-W_{2}\right)^{2}\right]
$$

is the "Triangle Function" (Heron's expression for the square of the area of the triangle in terms of the lengths of its sides) of $\mathrm{W}$, the invariant mass of the complete $n$ particle system and $W_{1}, W_{2}$ are the masses of the subsystems with $n_{1}, n_{2}$ particles. $\Theta(x)$ is the unit step function. The resulting phase space values for $n=1,2, \ldots 8$ are presented in table II below. For masses, energies and momenta we use the $\mathrm{GeV}$ unit throughout this paper. In particular, the dimensions of the LIPS for $n+1$ particles is $\mathrm{GeV}^{(2 n-2)}$. Following the convention of Perl[5] we do not include the $(2 \pi)^{-3}$ normalization factors for each final state particle in the phase space.

To estimate $\Gamma\left(\tau^{-} \rightarrow \pi^{+} \pi^{+} \pi^{+} \pi^{-} \pi^{-} \pi^{-} \pi^{-} \nu_{\tau}\right)$ we compare it with the decay into $\nu_{\tau}$ and five charged pions with a measured $\mathrm{BR} \sim 1 \%$ and a theoretical estimate analogous to equation 14: 
TABLE II: In this table we list the phase space for $\tau$ decays to a neutrino and $n$ charged pions.

$$
\begin{aligned}
& n \text { in } \tau \rightarrow n \pi \text { Phase Space } \\
& 1 \quad 1.56111 \\
& 2 \quad 3.10573 \\
& 3 \quad 1.75786 \\
& 4 \quad 0.383552 \\
& 5 \quad 0.0354896 \\
& 6 \quad 0.00139612 \\
& 7 \quad 2.17774 \times 10^{-5} \\
& 8 \quad 1.15161 \times 10^{-7} \\
& \Gamma\left(\tau^{-} \rightarrow \pi^{+} \pi^{+} \pi^{-} \pi^{-} \pi^{-} \nu_{\tau}\right)=\left(\frac{c_{5}}{5}\right)^{2} \frac{G_{F}^{2} m_{\tau}^{3} \bar{\Phi}\left(m_{\tau}, m_{\pi}, m_{\pi}, m_{\pi}, m_{\pi}, m_{\pi}, 0\right)}{\left(2 f_{\pi}\right)^{6}(2 \pi)^{18}}
\end{aligned}
$$

using equations 14 and 17:

$$
\begin{aligned}
\frac{B R\left(\tau^{-} \rightarrow \pi^{+} \pi^{+} \pi^{+} \pi^{-} \pi^{-} \pi^{-} \pi^{-} \nu_{\tau}\right)}{B R\left(\tau^{-} \rightarrow \pi^{+} \pi^{+} \pi^{-} \pi^{-} \pi^{-} \nu_{\tau}\right)} & =\frac{\bar{\Phi}\left(m_{\tau}, m_{\pi}, m_{\pi}, m_{\pi}, m_{\pi}, m_{\pi}, m_{\pi}, m_{\pi}, 0\right)}{(2 \pi)^{6}\left(2 f_{\pi}\right)^{4} \bar{\Phi}\left(m_{\tau}, m_{\pi}, m_{\pi}, m_{\pi}, m_{\pi}, m_{\pi}, 0\right)}\left(\frac{5 c_{7}}{7 c_{5}}\right)(18) \\
& =6 \times 10^{-6}\left(\frac{5 c_{7}}{7 c_{5}}\right)^{2}
\end{aligned}
$$

Using the $1 \%$ branching ratio into 5 charged pions we then have

$$
B R\left(\tau^{-} \rightarrow \pi^{+} \pi^{+} \pi^{+} \pi^{-} \pi^{-} \pi^{-} \pi^{-} \nu_{\tau}\right)=6 \times 10^{-8}\left(\frac{5 c_{7}}{7 c_{5}}\right)^{2}
$$

In Appendix I we find that $\left(5 c_{7} / 7 c_{5}\right)=1 / 30$ and thus equation 20 leads to a hopelessly small branching ratio $B R\left(\tau^{-} \rightarrow \pi^{+} \pi^{+} \pi^{+} \pi^{-} \pi^{-} \pi^{-} \pi^{-} \nu_{\tau}\right)=6 \times 10^{-11} \cdot[6]$

The chiral Lagrangian approach used here is justified all momenta in the problem are small. For the case at hand the total invariant mass of the seven pion hadronic system and masses of 3, 4, and 5 pion subsystems are typically high $(W \approx 1 \mathrm{GeV})$. Various resonances $a_{1}(1240) a_{2}(1320), \pi(1300)$ etc. can then appear in these subchannels. These resonances could significantly enhance certain rates and invalidate the local effective Lagrangian approach. 
We would like however to argue that while resonances typically dominate decays of the tau lepton (and also of charmed hadrons) into 2-4 particles, their effect on the multi-particle decays of interest with many soft slow pions may be minimal. The point is that these heavy resonances with prominent multiparticle decays are very broad, $\Gamma=300-400 \mathrm{MeV}$. These resonances also move rather slowly $(\beta<1 / 2)$ and the decay products of different resonances emerge within a common interaction range. Hence resonance (re-)formations and decays can, in the context of the present final state of many, highly overlapping, soft pions be subsumed into the local effective Lagrangian.

In the momentum space description the $n$-particle vertex is effectively local so long as the real off-shellness of the internal particles exchanged OR the imaginary part therof (viz. widths) are large in comparison with the final physical particle momenta.

The effect of resonances is likely to be stronger when fewer particles occur in the final state. Hence our value for the $7 \pi$ branching ratio obtained by treating both this and the $5 \pi$ decay by the same chiral Lagrangian and not accounting for possible resonance enhancement of the latter may well yield an overestimate of $B R\left(\tau \rightarrow 7 \pi \nu_{\tau}\right)$.

The situation is drastically different when the multipion final state is dominated by narrow resonances. This, in particular, is the case for the $\tau \rightarrow 6 \pi \nu_{\tau}$ recently observed by CLEO.[7] These observations are consistent with being of the form $\tau \rightarrow \eta 3 \pi \nu_{t} a u$ and $\tau \rightarrow \omega 3 p i \nu_{t} a u$ with subsequent decays of the $\eta$ and $\omega$ to $3 \pi$.

Low energy $\pi \pi$ scattering in general and scattering lengths in particular are studied in simpler settings such as $K_{l 4}$ decays or the $\pi N \rightarrow \pi \pi N$ reaction in a region dominated by an almost real pion exchange. The resulting S-wave scattering lengths

$$
a(I=0) \approx 0.22 / m_{\pi} \quad \text { and } \quad a(I=2) \approx-(0.02-0.08) / m_{\pi}
$$

are relatively small in agreement with various theoretical considerations [8], [9].

The many-pion final states of interest could be particularly sensitive to the threshold $\pi \pi$ interaction. To analyze this we use a non-relativistic description. The total interaction energy in the $n$ pion state, when all pions are within interaction range, increases quadratically with $n$ :

$$
U=\sum V_{i j}\left(r_{i}-r_{j}\right) \approx n(n-1) / 2<V>
$$


where $\langle V\rangle$ indicates the expectation value of an attractive interaction.

As we shortly indicate, this interaction is attractive. If the pions can be treated as pointlike elementary bosons this will always lead eventually to condensation. All pions can then be put into a common state - the (S-wave) ground state inside a spherical cavity of radius $r$ smaller than the $\pi-\pi$ interaction range which is denoted by $r$. The kinetic energy $T=n \hbar^{2} / 2 m r^{2}$ (or $n \hbar / r$ ) which is linear in $n$ is less than the attractive potential energy of equation 22 .

$$
n>1+\frac{2<T>}{|<V>|}
$$

For $n$ larger than $n_{c}$ defined by

$$
n_{c}=1+\frac{2 m_{\pi}}{<V>}
$$

the interaction energy exceeds even the rest mass of the pions. The description in terms of pions ceases then to be useful and the system is better described by the $\phi$ (or $\phi^{2}$ ) field. In particular the rate for producing more than $n_{c}$ pions in $\tau \rightarrow n \pi+\nu_{\tau}$ could be dramatically enhanced.

As we will argue next, the requisite $n_{c}$ is far higher than the number of pions in a single cluster produced in tau decays or $\bar{p} p$ annihilations at rest.

Let $n=2 k+1$ so that the $\tau^{+}$decays into $k+1$ positive and $k$ negative pions. We have $k(k+1) / 2++$ pairs and $\mathrm{k}(\mathrm{k}-1) / 2--$ pairs, a total of $k^{2}$ pairs which are in a pure $\mathrm{I}=2$ state, and $k(k+1)+-$ pairs which constitute $\mathrm{I}=0$ and $\mathrm{I}=2$ states in a 2:1 ratio. (Since all pairs are in relative $\mathrm{S}$-wave states, Bose statistics forbids any $\mathrm{I}=1$ states). The effective $\mathrm{S}$-wave scattering length averaged over all $(2 k+1) k$ possible pairs is therefore:

$$
\begin{aligned}
a_{\mathrm{eff}} & =\frac{\left[k^{2}+k(k+1) / 3\right] a(I=2)+(2 / 3) k(k+1) a(I=0)}{(2 k+1) k} \\
& =\frac{(4 k+1) a(I=2)+(2 k+2) a(I=0)}{3(2 k+1)}
\end{aligned}
$$

For large $k$ we simply have

$$
a_{\mathrm{eff}}=\frac{2 a(I=2)}{3}+\frac{a(I=0)}{3} \approx(0.06-0.02) m_{\pi}^{-1}
$$


and in particular, as stated above, is attractive.

Let us next proceed to a rough estimate of the critical number of particles which is the threshold for the onset of condensation from either equation 23 or equation 24 above. For simplicity the $\pi-\pi$ interaction is approximated by a square well of depth $-V_{0}$ and radius $r_{0}$. Elementary considerations then yield $a=\tan \left(q r_{0}\right) / q-r_{0}$, where $q=\sqrt{m_{\pi} V_{0}}$.

Since there is no near threshold $\pi-\pi$ bound state and $q r_{0} \leq 1$ we expand $\tan \left(q r_{0}\right)$, finding

$$
a \approx \frac{\left(q r_{0}\right)^{3}}{3 q}=\frac{1}{3} m_{\pi} V_{0} r_{0}^{3}
$$

We next used equation 28 and estimates of the expectation values $\langle T\rangle$ and $\langle V\rangle$ pertinent to this specific square well model to obtain from equation 23:

$$
n-1>\frac{(\pi)^{2} r_{0}}{3 a}=16-48
$$

The actual numbers on the right hand side of equation 29 were obtained for $r_{0}=1 /\left(3 m_{\pi}\right)$ and the range of $a_{e f f}$ in equation 27. (We note that since for this $r_{0}$ value $<T>\approx 1 / r_{0} \geq m_{\pi}$ equation 23 implies equation 24).

Even if we use $r_{0}=1 /\left(5 m_{\pi}\right)$ corresponding to an exchange of a " $\sigma$ " particle of mass 700 $\mathrm{MeV}, n$ is still in the range $10-30$.

The above considerations strongly suggest that for the 7-pion final state of interest any condensate enhancements are most unlikely and our above estimates of rates should stand.

\section{ACKNOWLEDGEMENTS}

We would like to thank Silas Beans for his help and advice regarding the chiral Lagrangian calculations presented in the Appendix and to Manoj Banerjee for his interest. S. N. thanks the Israeli National Science Foundation (grant 561/99) and M. V. P. thanks the U.S. Department of Energy. 


\section{APPENDIX I}

We will estimate the decay rate $\Gamma\left(\tau^{-} \rightarrow \pi^{+} \pi^{+} \pi^{+} \pi^{-} \pi^{-} \pi^{-} \pi^{-} \nu_{\tau}\right)$ using the leading order axial vector contribution in chiral perturbation theory. The leading order chiral Lagrangian is given by

$$
\mathcal{L}=\frac{1}{4} f_{\pi}^{2} \operatorname{Tr}\left(\partial_{\mu} \Sigma^{\dagger} \partial_{\mu} \Sigma\right)
$$

The field $\Sigma$ transforms linearly with respect to $S U(2)_{L} \times S U(2)_{R}: \Sigma \rightarrow L \Sigma R^{\dagger}$ where $L, R$ is an element of $S U(2)_{L, R}$. A convenient parameterization of $\Sigma$ is

$$
\Sigma=\exp \left(\frac{i \phi_{a} \tau_{a}}{f_{\pi}}\right)
$$

where $f_{\pi}=93 \mathrm{MeV}$ and the $\tau_{a}$ are the Pauli matrices. The Noether current associated with $S U(2)_{L}$ is

$$
J_{L a}^{\mu}=i \frac{1}{4} f_{\pi}^{2} \operatorname{Tr}\left(\Sigma^{\dagger} \tau_{a} \partial^{\mu} \Sigma\right) .
$$

With the help of a useful mathematical formula [10] one finds

$$
J_{L a}^{\mu}=-\frac{1}{4} f_{\pi} \int_{0}^{1} d s \operatorname{Tr}\left[\tau_{a}\left(\partial^{\mu} \tilde{\phi}+\frac{i s}{f_{\pi}}\left[\tilde{\phi}, \partial^{\mu} \tilde{\phi}\right]+\frac{1}{2 !}\left(\frac{i s}{f_{\pi}}\right)^{2}\left[\tilde{\phi},\left[\tilde{\phi}, \partial^{\mu} \tilde{\phi}\right]\right]+\mathcal{O}\left(s^{3}\right)\right)\right]
$$

where $\tilde{\phi} \equiv \phi_{a} \tau_{a}$. The $S U(2)_{R}$ current can then be obtained by switching the signs of all the odd terms in the pion field. The axial vector current is

$$
A_{a}^{\mu}=-\frac{1}{2} f_{\pi} \int_{0}^{1} d s \operatorname{Tr}\left[\tau_{a}\left(\partial^{\mu} \tilde{\phi}+\frac{1}{2 !}\left(\frac{i s}{f_{\pi}}\right)^{2}\left[\tilde{\phi},\left[\tilde{\phi}, \partial^{\mu} \tilde{\phi}\right]\right]+\mathcal{O}\left(s^{4}\right)\right)\right]
$$

It is then straightforward to find

$$
A_{a}^{\mu}(n)=-\frac{1}{2} f_{\pi} \frac{1}{n !}\left(\frac{i}{f_{\pi}}\right)^{n-1} \operatorname{Tr}\left(\tau_{a}\left[\tilde{\phi}, \ldots,\left[\tilde{\phi},\left[\tilde{\phi},\left[\tilde{\phi}, \partial^{\mu} \tilde{\phi}\right], \ldots,\right]\right]\right]\right) .
$$

where $n$ is the number (odd) of pions. For instance,

$$
A_{a}^{\mu}(1)=-\frac{1}{2} f_{\pi} \operatorname{Tr}\left(\tau_{a} \partial^{\mu} \tilde{\phi}\right)=-f_{\pi} \partial^{\mu} \phi_{a}
$$




$$
\begin{gathered}
A_{a}^{\mu}(3)=\frac{1}{12 f_{\pi}} \operatorname{Tr}\left(\tau_{a}\left[\tilde{\phi},\left[\tilde{\phi}, \partial^{\mu} \tilde{\phi}\right]\right]\right) ; \\
A_{a}^{\mu}(5)=-\frac{1}{240 f_{\pi}^{3}} \operatorname{Tr}\left(\tau_{a}\left[\tilde{\phi},\left[\tilde{\phi},\left[\tilde{\phi},\left[\tilde{\phi}, \partial^{\mu} \tilde{\phi}\right]\right]\right]\right]\right) ; \\
A_{a}^{\mu}(7)=\frac{1}{10080 f_{\pi}^{5}} \operatorname{Tr}\left(\tau_{a}\left[\tilde{\phi},\left[\tilde{\phi},\left[\tilde{\phi},\left[\tilde{\phi},\left[\tilde{\phi},\left[\tilde{\phi}, \partial^{\mu} \tilde{\phi}\right]\right]\right]\right]\right]\right]\right) .
\end{gathered}
$$

Using $\left[\tau_{i}, \tau_{j}\right]=\epsilon_{i j k} \tau_{k}$ we can write

$$
A_{a}^{\mu}(7)=\frac{1}{10080 f_{\pi}^{5}} \epsilon_{a b c} \epsilon_{e d c} \epsilon_{f g e} \epsilon_{i h f} \epsilon_{k j i} \epsilon_{m l k} \phi_{l} \phi_{j} \phi_{h} \phi_{g} \phi_{d} \phi_{b} \partial_{\mu} \phi_{a}
$$

By pairwise contraction of $\epsilon$ symbols we can reduce the expression into the desired $c(7)(\phi$. $\phi)^{3} \partial_{\mu} \phi$ form. Likewise we generate from $A_{\mu}(5)$ of Eq. (38) above an analogous $c_{5}(\phi \cdot \phi)^{2} \partial_{\mu} \phi$ term. We find that $c(7)=2^{3} / 10080$ and $c(5)=2^{2} / 240$. Hence, in particular, $5 c(7) / 7 c(5)=$ $10 / 294 \approx 1 / 30$.

Actually all currents $A(1)-A(7)$ contribute to the decay $\tau^{-} \rightarrow \pi^{+} \pi^{+} \pi^{+} \pi^{-} \pi^{-} \pi^{-} \pi^{-} \nu_{\tau}$ at leading order in chiral perturbation theory. For instance, $A_{a}^{\mu}(5)$ contributes with one of the pions going to three pions through the 4-point interaction contained in Eq. (30). In order to evaluate these we need more involved calculations. Thus the last contribution is the integral over $\sigma=\sqrt{s(678)}$ of the product of the $A(5)$ amplitude with one pion off-shell at $\sigma$ and the $\pi \pi$ scattering with one pion off-shell at the same $\sigma$. All these contributions should be comparable and we do not expect these to drastically change our estimate of the $c(7) / c(5)$ ratio.

[1] Note that the spectator and annihilation decays correspond (at the quark level) to different two and four quark states. The amplitudes for annihilation and direct $Q$ decay interfere in specific (hadronic) decay channels, but not in the inclusive rates. Hence one adds the two contributions in the total decay rate.

[2] Particle Data Group, D.E. Groom et al., "The Review of Particle Physics", Eur. Phys. J. C15 (2000) 1 and references therein.

[3] J. Rosner, private communication, attributed to D. Jovanovic. 
[4] R. Fischer, J. Wess and F. Wagner, Z. Phys C3 313 (1979).

[5] M. L. Perl, "High Energy Hadron Physics", John Wiley \& Sons, 1974.

[6] If we were exactly at threshold, with all the pions in a common S-wave state, the rate is enhanced by a "Bose-Einstein" factor $n_{\pi^{+}} ! n_{\pi^{-}}$!. Since the pions in the $7 \pi$ decay are slower than those in the $5 \pi$ decay the enhancement factor there is closer to the expected value of $4 ! \times 3 !=144$ than the one in the 5 pion decay, $3 ! \times 2 !=24$. However, even in the unlikely case where this enhancement is fully operative in $\tau \rightarrow 7 \pi \nu_{\tau}$ and not at all in $\tau \rightarrow 5 \pi \nu_{\tau}$, the 7 pion $B R\left(\tau \rightarrow 7 \pi \nu_{\tau}\right)=6 \times 10^{-9}$ is still $\sim 400$ times smaller than the present upper bound.

[7] A. Anastassov et al., Phys. Rev. Lett. 864467 (2001).

[8] J. F. Donoghue, E. Golowich and B. R. Holstein, in "Dynamics of the Standard Model", Cambridge University Press, Cambridge, UK (1992).

[9] H. Leutwyler, "Theoretical Chiral Dynamics" to be published in Proceedings of "Chiral Dynamics 2000: Theory and Experiment", Jefferson Laboratory (2000).

[10] H. Georgi, Weak Interactions and Modern Particle Theory, (Benjamin/Cummings, Menlo Park, CA, 1984), p77. 